\title{
Evolution of Near-Surface Deformed Layers on AA3104 Aluminium Alloy
}

\author{
Kai $\mathrm{Li}^{1, \mathrm{a}}$, Xiaorong Zhou ${ }^{1, \mathrm{~b}}$, George Thompson ${ }^{1, \mathrm{c}}$, John Hunter ${ }^{2, \mathrm{~d}}$ \\ and Yudie Yuan ${ }^{2, e}$ \\ ${ }^{1}$ School of Materials, The University of Manchester, Manchester, M13 9PL, UK \\ ${ }^{2}$ Novelis Global Research and Technology Center, Kennesaw, GA 30144, USA \\ akai.li-2@postgrad.manchester.ac.uk, bxiaorong.zhou@manchester.ac.uk, \\ cgeorge.thompson@manchester.ac.uk, ${ }^{\mathrm{c}}$ john.hunter@novelis.com, ${ }^{\mathrm{e}}$ yudie.yuan@novelis.com
}

Keywords: Deformed layer, Aluminium alloy, Rolling, Ultrafine grains, Oxide particles

\begin{abstract}
The present work studied the microstructure of near-surface deformed layers and their evolution from the transfer slab to the cold rolled final gauge sheet of an AA3104 aluminium alloy. Electron microscopy of ultramicrotomed cross-sections revealed two types of near-surface deformed layers, i.e. type $\mathrm{A}$ and type $\mathrm{B}$, both with different microstructures to the underlying bulk alloy. A typical feature of the deformed layers is the nano-sized ultrafine grains, with diameters $\leq 200 \mathrm{~nm}$ for the type A and $\leq 500 \mathrm{~nm}$ for the type B deformed layer. Electron energy loss spectroscopy (EELS) indicated that oxide particles are present along grain boundaries within the type A deformed layer, while the type B deformed layer is free of oxide particles.

The type A deformed layer is mainly generated at elevated temperatures during the early stages of hot rolling. Its thickness is non-uniform across the surface, with a maximum of $\sim 4 \mu \mathrm{m}$ on the transfer slab, $\sim 1 \mu \mathrm{m}$ on the re-roll gauge sheet and $\sim 0.8 \mu \mathrm{m}$ on the final gauge sheet. While the surface of the hot rolled sheet is mainly covered by the type A deformed layer, the surface undergone cold rolling is alternately covered by the type A and the type B deformed layers.
\end{abstract}

\section{Introduction}

The surface/near-surface region of aluminium sheet is subjected to enhanced shear strains during rolling, resulting in the formation of near-surface deformed layers with different microstructures to the underlying bulk alloy [1-5]. The deformed layer has a significant influence on the optical properties, electrochemical response and corrosion behaviour, and the joining and welding behaviour $[2,5,6]$. Therefore, understanding the evolution of the deformed layers through rolling processes is of considerable interest to the aluminium industry. However, the mechanisms and processes involved in the formation of the deformed layers on rolled aluminium alloys are still the subject of discussion.

In the present study, the structure, thickness, uniformity and composition of the deformed layers have been characterized by scanning and transmission electron microscopies with the aim of understanding the evolution of deformed layers through hot and cold rolling. A further aim is to achieve a thorough understanding of the precise formation mechanisms of the deformed layers on rolled aluminium alloys.

\section{Experimental}

AA3104 aluminium alloy (Si 0.2 wt.\%, Fe 0.4 wt.\%, Cu 0.2 wt.\%, Mn 0.9 wt.\%, Mg 1.2 wt.\%, Zn 0.07 wt. \%, Cr 0.03 wt. $\%$, Ti 0.01 wt.\%, Al balance) was studied in the as-rolled condition. The evolution of the near-surface microstructures was assessed by examining samples taken after sequential stages of hot and cold rolling, e.g. the transfer slab taken from the reverse breakdown mill, the re-roll gauge sheet taken from the tandem mills, and the final gauge sheet taken after a 3-pass cold rolling process.

Characterization of the near-surface deformed layers and the underlying bulk alloy was carried out using scanning electron microscopy (SEM) and transmission electron microscopy (TEM). Specimens were prepared by ultramicrotomy, using a diamond knife on a Leica Ultracut ultramicrotome. SEM 
imaging was carried out on the stub, while TEM and electron energy loss spectroscopy (EELS) were undertaken on the electron transparent sections cut from the stub, with a nominal thickness of $15 \mathrm{~nm}$.

\section{Results}

Microstructure of the Near-Surface Deformed Layer. Fig. 1 displays scanning and transmission electron micrographs of the surface of a cold rolled AA3104 aluminium alloy and the corresponding ultramicrotomed sections of the surface/near-surface region. Fig. 1a reveals a typical surface appearance of the cold rolled aluminium alloy, i.e. with imprinted surface morphology of the work roll and surface cracks passing transverse to the rolling direction. Fig. 1b shows a scanning electron micrograph of an ultramicrotomed stub; the cross-section was cut along the dashed line indicated in Fig. 1a. A layer with a different microstructural feature to the underlying bulk alloy is clearly evident in the near-surface region. This layer is characterized by dark spots and the thickness is not uniform across the surface, with a maximum thickness of $800 \mathrm{~nm}$ in the central region and a thickness less than $200 \mathrm{~nm}$ on the right-hand side region. However, the layer is not present across the entire surface, e.g. the layer is absent on the left-hand side of the near-surface region of the specimen. A transmission electron micrograph of the region indicated by frame 2 in Fig. 1b is shown in Fig. 1d. It exhibits the presence of a near-surface deformed layer, characterized by ultrafine grains, approximately 50-200 $\mathrm{nm}$ in diameter, which are clearly revealed in the inset in Fig. 1d. Further, a high population density of particles, with sizes less than $50 \mathrm{~nm}$, are present within the deformed layer. Fig. 1c displays a transmission electron micrograph of frame 1 region indicated in Fig. 1b. Interestingly, it also reveals a near-surface deformed layer characterized by very fine grains, approximately $200-500 \mathrm{~nm}$ in diameter, as exhibited by the inset in Fig. 1c. Although the grains are slightly coarser than those revealed in Fig. 1d, they are much finer compared with the underlying bulk alloy, where the grain size ranges between 1 to $2 \mu \mathrm{m}$. Nevertheless, the particles shown in Fig. 1d are not present in this type of deformed layer.
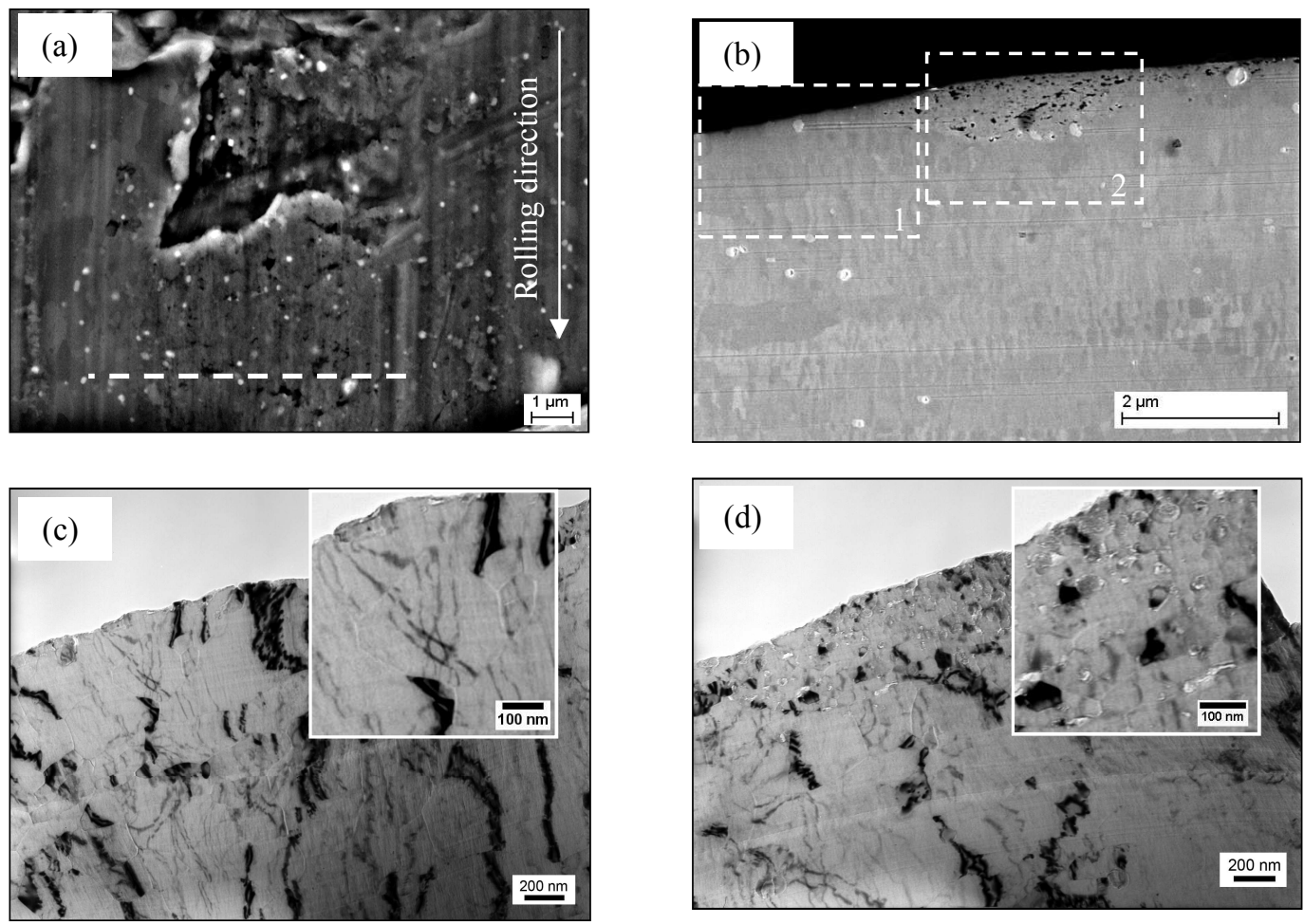

Fig. 1. Near-surface deformed layers on an AA3104 aluminium alloy (a) SEM image of the alloy surface, (b) SEM image of an ultramicrotomed stub, the cross-section was cut along the dashed line in (a), (c) TEM image of frame 1 in (b), (d) TEM image of frame 2 in (b). 
The near-surface microstructure of the cold rolled AA3014 aluminium alloy was further examined by transmission electron microscopy and electron energy loss spectroscopy mapping, as illustrated in Fig. 2. A deformed layer is present within the near-surface region, characterized by ultrafine grains and grain boundaries decorated by nano-sized particles. Its maximum thickness is approximately $500 \mathrm{~nm}$. Electron energy loss spectroscopy mapping revealed that the nano-sized particles are enriched with oxygen, as shown in Fig. 2b. Correspondingly, the regions enriched with oxygen contain a decreased yield of aluminium compared with the matrix, indicating that they are oxides, which are embedded into the near-surface region during rolling $[1,3,4]$. Generally, the oxides should also enrich with magnesium due to its preferential oxidation and outward diffusion in magnesium-containing aluminium alloys $[1,4]$. However, as shown in Fig. 2d, there is little contrast showing the distribution of magnesium corresponding to the particles. This might be attributed to the preferential dissolution of magnesium in the water bath during ultramicrotomy.
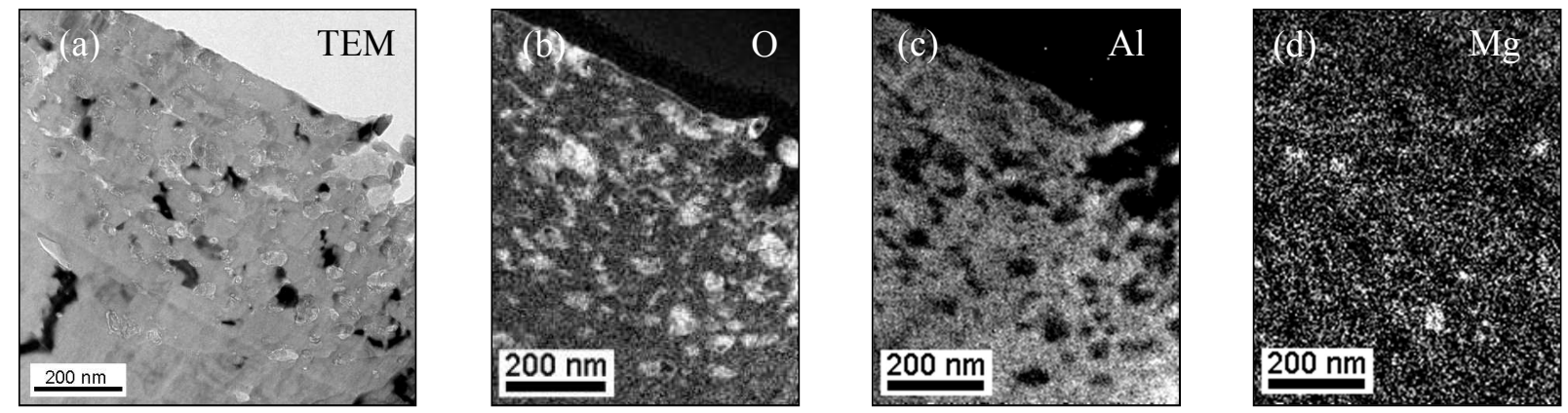

Fig. 2 Ultramicrotomed section of the surface/near-surface region of an AA3104 aluminium alloy: (a) TEM image; (b) EELS map of oxygen; (c) EELS map of aluminium; (d) EELS map of magnesium.

In order to distinguish the two types of near-surface deformed layers revealed in Fig. 1c\&d, the one characterized by ultrafine grains and grain boundaries decorated by oxide particles is termed type $\mathrm{A}$, and the one which is also characterized by very fine grains but with grain boundaries free of oxide particles is termed type B. Since oxygen is a lighter element than aluminium, regions enriched with oxygen, i.e. the oxide particles in this case, appear darker than the surrounding aluminium matrix in the scanning electron micrograph. This explains why the type A deformed layer could be revealed in Fig. 1b, but the type B deformed layer could not be displayed.

Evolution Through Rolling Processes. Fig. 3 exhibits transmission electron micrographs of ultramicrotomed sections of the surface/near-surface regions of an AA3104 aluminium alloy, with the samples taken after sequential stages of hot and cold rolling, displaying the evolution of the near-surface deformed layers. As shown in Fig. 3a, a type A deformed layer, characterized by ultrafine grains and oxide particles, with a maximum thickness of approximately $4 \mu \mathrm{m}$, is evident in the near-surface region of the transfer slab taken from the reverse breakdown mill. Its thickness is not uniform across the surface, but the surface is mainly covered with the type A deformed layer.

Fig. $3 \mathrm{~b}$ reveals the type A deformed layer in the near-surface region of the re-roll gauge sheet taken from tandem mills. The deformed layer, again, is characterized by ultrafine grains and oxide particles but has a decreased thickness, with a maximum of approximately $1 \mu \mathrm{m}$. The thickness is not uniform across the surface, and the surface is also mainly covered with the type A deformed layer.

On the final gauge sheet that had 3 cold rolling passes, the type A deformed layer is still present in the near-surface region but the thickness is further decreased to less than $800 \mathrm{~nm}$, as illustrated in Fig. 3c. It should be noted that this specimen was sectioned along the rolling direction while all others were sectioned transverse to rolling direction. The ultrafine grains of the type A deformed layer are slightly elongated in the rolling direction. The underlying grains are elongated in the rolling direction as well. Differently, the surface of the final gauge sheet is not mainly covered with the type A deformed layer, as indicated by the dashed line in Fig. 3c. A type B deformed layer, also characterized by very fine grains, but with grain boundaries free of oxide particles, is present within the near-surface region as well. The surface is alternately covered by the type A and the type B deformed layers. 


\section{Discussion}

Formation of the Near-Surface Deformed Layer. The results presented indicate that, during rolling processes of AA3104 aluminium alloy, near-surface deformed layers were generated with different microstructures to the underlying bulk alloy. Two types of deformed layers were observed. The type A deformed layer was characterized by ultrafine grains and with grain boundaries decorated by oxide particles, as shown in Figs. 1d\&2. The type B deformed layer was also characterized by very fine grains, but with grain boundaries free of oxide particles, as shown in Fig. 1c.
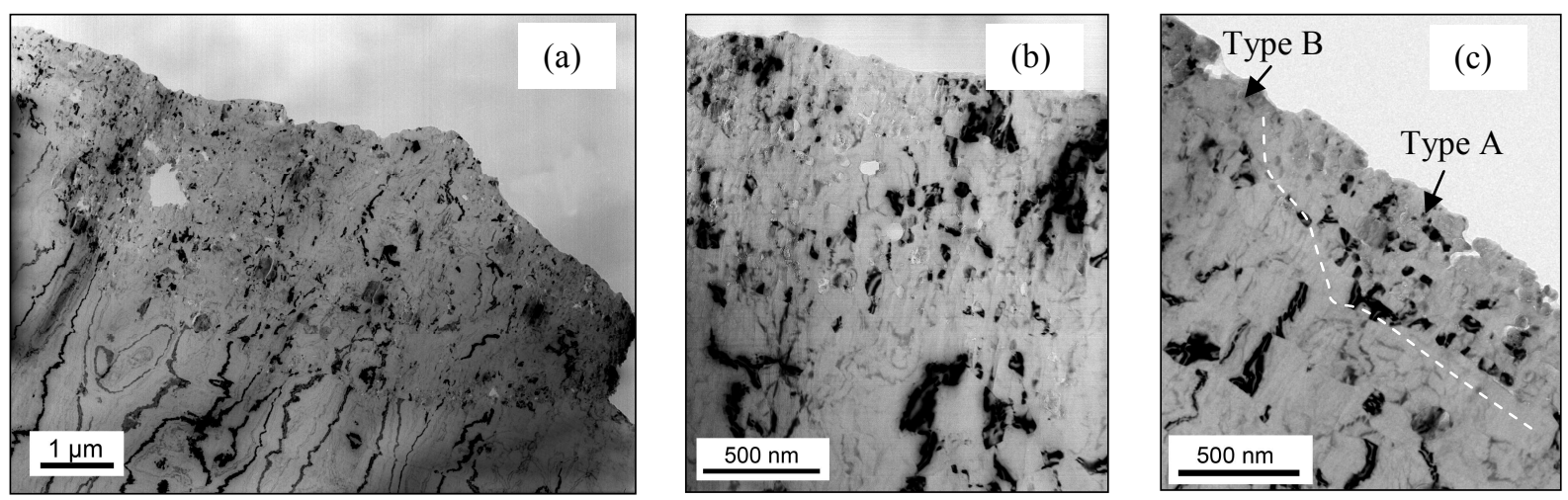

Fig. 3. TEM images of ultramicrotomed sections of the surface/near-surface region of an AA3104 aluminium alloy (a) after the reverse breakdown mill, (b) after the tandem mills, (c) after a 3-pass cold rolling process.

A high level of plastic strain is introduced into the surface/near-surface region of the work-piece by mutual sliding contact during rolling [1,4]. Yarita et al. [7] suggested that the shear strain in the roll bite is highest on the surface and decreases gradually from the surface to the centre of the bulk material. Further, the shear strain undergoes a reversal in the neutral plane of roll bite. During deformation of aluminium alloys at elevated temperatures, subgrain formation is promoted by dynamic recovery, which is attributed to the high stacking fault energies. As a result, grain boundaries will develop serrations since subgrain boundaries form. Due to the severe plastic surface strain during rolling, the original grains in the surface/near-surface region become flattened and will thin to a point that their thickness is in the order of about twice the subgrain size. Pinch-off and annihilation of the serrated boundaries will occur and result in a marked microstructure refinement. This process is referred as geometric dynamic recrystallization, which is the origin for the formation of the near-surface deformed layers characterized by ultrafine grains [3,4,8-10].

In addition to geometric dynamic recrystallization, incorporation of oxides into the near-surface region is the second origin for the formation of the type A deformed layer. For magnesium-containing aluminium alloys, magnesium is readily diffused outwards and is oxidized to form $\mathrm{MgO}$ through direct oxidation or by reduction of amorphous alumina, leading to a surface oxide layer that consists of $\mathrm{Al}_{2} \mathrm{O}_{3}, \mathrm{MgO}$ and/or spinel $\mathrm{MgAl}_{2} \mathrm{O}_{4}$ [1,4]. The surface oxide layer formed at earlier stages could be broken-up during subsequent rolling due to its brittleness and a lower surface ductility caused by temperature decrease. Combined with the severe surface shear strain and its reversal in the neutral plane of roll bite, the oxides tend to be embedded into the near-surface region [1,4]. Furthermore, thin alloy layers could be transferred to the roll during rolling. They are oxidized and can be transferred back to the alloy surface $[3,4]$. Both of them contribute to the formation of the type A deformed layer.

Through rolling processes, the surface oxide layer is progressively thinned due to the extension of aluminium sheet and a decrease in the oxidation rate arises from surface cooling. Thus, the incorporation of oxides into the near-surface region, and also the formation of the type A deformed layer, is more prominent at elevated temperatures, i.e. early stages of hot rolling. During subsequent rolling, the thickness of the type A deformed layer is gradually reduced since it is distributed over a larger surface area with the extension of the alloy surface [1,4]. Conversely, the type B deformed layer is generated at both hot and cold rolling stages, induced by the severe plastic surface strain [4]. 
Stability of the Near-surface Deformed Layer. Humphreys et al. [9] reported that the main criteria for the formation of a sub-micron grain structure is the generation of a sufficiently large fraction (greater than 0.7 ) of high angle grain boundaries during the deformation process. This is consistent with the present study. Further, the high population density of oxide particles present at grain boundaries within the type A deformed layer may retard the motion of grain boundaries by the Zener drag process and provide sites where grain boundaries are pinned, thus preventing any significant coarsening $[3,4]$.

For the type B deformed layer, since it is free of oxide particles and thus without the Zener pinning effect, slight grain growth may proceed at subsequent rolling stages, even during cold rolling where surface temperatures up to $150{ }^{\circ} \mathrm{C}$ are possible [11]. This is consistent with the result that the grains within the type B deformed layer are slightly coarser than those within the type A deformed layer.

\section{Conclusions}

(1) Two types of near-surface deformed layers are present on AA3104 aluminium alloy. Both of them are characterized by ultrafine grains, with diameters $\leq 200 \mathrm{~nm}$ for the type A and $\leq 500 \mathrm{~nm}$ for the type B deformed layers. Oxide particles are decorated along grain boundaries within the type A deformed layer, while the type B deformed layer is free of oxide particles.

(2) The type A deformed layer is mainly formed at elevated temperatures during the early stages of hot rolling. Its thickness is non-uniform across the surface, with a maximum of $\sim 4 \mu \mathrm{m}$ on the transfer slab, $\sim 1 \mu \mathrm{m}$ on the re-roll gauge sheet and $\sim 0.8 \mu \mathrm{m}$ on the final gauge sheet. Conversely, the type $\mathrm{B}$ deformed layer is generated at both hot and cold rolling stages.

(3) The surface of the hot rolled sheet is mainly covered with the type A deformed layer. But the surface of the cold rolled sheet is alternately covered by the type A and the type B deformed layers.

\section{Acknowledgements}

The authors wish to thank Novelis and the Engineering and Physical Sciences Research Council for provision of financial support through supported studentships and the LATEST2 Programme Grant. The financial support of the China Scholarship Council is also gratefully acknowledged.

\section{References}

[1] M. Fishkis, J.C. Lin, Wear 206 (1997) 156-170.

[2] Y. Liu, et al., Acta Mater. 55 (2007) 353-360.

[3] Y. Liu, et al., Surf. Interface Anal. 42 (2010) 180-184.

[4] X. Zhou, Y. Liu, G.E. Thompson, G.M. Scamans, P. Skeldon, J.A. Hunter, Metall. Mater. Trans. A 42 (2010) 1373-1385.

[5] G.M. Scamans, M.F. Frolish, W.M. Rainforth, Z. Zhou, Y. Liu, X. Zhou, G.E. Thompson, Surf. Interface Anal. 42 (2010) 175-179.

[6] Y. Liu, et al., Surf. Interface Anal. 42 (2010) 185-188.

[7] I. Yarita, R. Mallett, E. Lee, Steel Res. 56 (1985) 255-259.

[8] F.J. Humphreys, M. Hatherly, Recrystallization and Related Annealing Phenomena, second ed., Elsevier, Oxford, 2004.

[9] F.J. Humphreys, et al., Phil. Trans. R. Soc. London Ser. A 357 (1999) 1663-1681.

[10] R.D. Doherty, et al., Mater. Sci. Eng. A 238 (1997) 219-274.

[11] D.G. Altenpohl, Aluminium: Technology, Applications and Environment, 6th ed., Aluminium Association, Washinton, D.C., 1998. 